
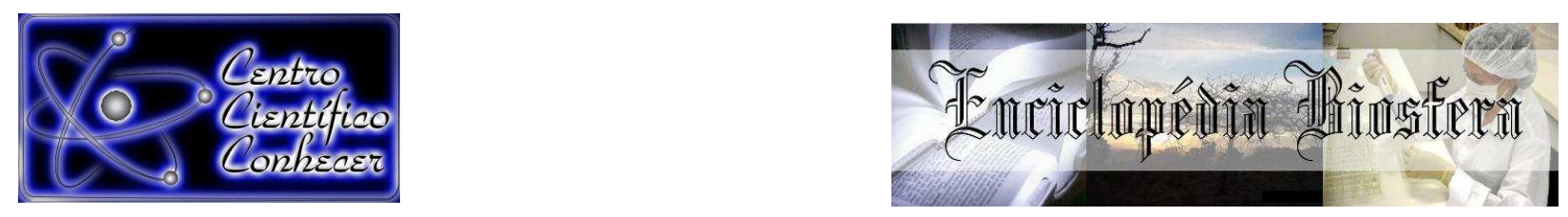

\title{
POTENCIALIDADE DO USO DE MÉTODO MATEMÁTICO PARA PREVISÃO DE MARÉ ASTRONÔMICA NO PORTO DA CIDADE DE BELÉM-PA
}

Mayke Feitosa Progênio1, Felipe Antonio Melo da Costa Filho², Raimundo Nonato da Silva Barbosa Junior ${ }^{3}$, Marcelo José Raiol Souza ${ }^{4}$, Luciana Leal Pimentel Oliveira ${ }^{5}$

${ }^{1}$ Graduando em Engenharia Ambiental - UEPA - CCNT Belém - Parámaykefeitosa@gmail.com

2 Graduando em Engenharia Ambiental - UEPA - CCNT

${ }^{3}$ Graduando em Engenharia Ambiental - UEPA - CCNT

${ }^{4}$ Professor Doutor na Universidade do Estado do Pará - UEPA

${ }^{5}$ Ms. em Engenharia Civil, Pós-Graduação em Engenharia Civil /ITEC/UFPA

Recebido em: 08/04/2017 - Aprovado em: 10/06/2017 - Publicado em: 20/06/2017 DOI: 10.18677/EnciBio_2017A155

\begin{abstract}
Este trabalho objetivou analisar a potencialidade do uso do método matemático chamado de Interpolação Cúbica Hermitiana para calcular a altura de maré astronômica no porto do munícipio de Belém-PA. Os polinômios interpoladores foram implementados em um código desenvolvido em linguagem FORTRAN 2003. Os dados de entrada no código foram retirados da Tábua de maré fornecida pela diretoria de Hidrografia e Navegação da Marinha do Brasil. Medições experimentais da altura de maré no porto juntamente com simulação da mesma no software SisBAHIA foram conduzidas com intuito de analisar a potencialidade do uso do método. Uma comparação entre os resultados obtidos pela interpolação, o experimento e o SisBAHIA mostraram que a Interpolação Cúbica Hermitiana consegue prever alturas de maré satisfatoriamente, tanto no período chuvoso quanto não chuvoso da região. Os resultados obtidos mostraram também que a Interpolação gera dados simulados mais próximos da altura de maré que o SisBaHiA. Uma análise de erro relativo dos resultados foi realizada e o erro médio obtido pela utilização da Interpolação foi de $21,6 \%$, demonstrando a potencialidade em cálculo de previsão de maré.
\end{abstract}

RESUMO

PALAVRAS-CHAVE: erro, interpolação, maré. 


\title{
POTENTIALITY OF A MATHEMATICAL METHOD FOR ASTRONOMICAL TIDE FORECAST IN THE PORT OF BELÉM-PA
}

\begin{abstract}
This study aimed to analyze the potential of the use of the mathematical method called Hermitian Interpolation Cubic to calculate the astronomical tide height in the municipality of the port of Belém-PA. The interpolating polynomials are implemented in a code developed in FORTRAN 2003 language. The input data in the code were removed from the tide board provided by the Board of Hydrography and Navigation of the Brazil Navy. Experimental tide height measurements at the port with the same simulation in SisBAHIA software were conducted in order to examine the potential using this method. A comparison between the results obtained by interpolating the experiment and SisBAHIA, shown that Cubic Interpolation Hermitian can predict tide heights satisfactorily, both in the rainy season as not rainy season. The results also showed that generates interpolation data closest simulated tide height that SisBaHiA. An error analysis on the results was performed and the average error obtained by use of interpolation was $21.6 \%$, demonstrating its potentiality to tide prediction calculation.
\end{abstract}

KEYWORDS: error, interpolation, tide.

\section{INTRODUÇÃO}

A maré é definida como a oscilação vertical da superfície do mar sobre a Terra, representa a energia dissipada pelos movimentos dos mares, geradas principalmente, pela atuação das forças gravitacionais da lua e do sol sobre diversos pontos do planeta (CLARK, 2007; OLIVEIRA et al., 2015). As subidas e descidas do nível do mar são denominadas respectivamente de enchente e vazante; quando esta se encontra na máxima elevação de maré é chamada de preamar (PM) e na mínima elevação é chamada de baixamar (BM). Existem tipos de marés que se referem à forma e característica de subida e descida e podem ser agrupadas em três classes: semidiurna, diurna e mista (CHARLIER \& FINKL, 2009; ARAQUISTAIN, 2010). No Brasil, as marés em geral têm comportamento semidiurno desde Vitória no Espírito Santo, até o litoral norte do país (LEITE NETO et al., 2015).

Os dados de elevação e abaixamento da maré são divulgados pela Marinha do Brasil através da DHN (Diretoria de Hidrografia e Navegação). Esses dados são disponibilizados através de tábuas onde são apresentados os valores máximos e mínimos das alturas de maré. Atualmente, a DHN divulga as previsões para 43 portos nacionais, duas ilhas oceânicas, duas ilhas costeiras, quatro barras, um fundeadouro, um atracadouro da costa do Brasil e um fundeadouro na Estação Antártica Comandante Ferraz, totalizando 54 locais (DHN, 2016).

Os parágrafos anteriores demonstram que os dados de altura de marés alta e baixa são vitais, principalmente, para a navegação e também para atividades que dependem destas medidas. Isso posto, o principal objetivo deste trabalho foi analisar a potencialidade de uso de um método matemático alternativo para o cálculo dos valores instantâneos das alturas de maré, conhecido como Interpolação Cúbica Hermitiana (ICH) (CUOMO et al., 2015), no porto da cidade de Belém-PA e em seguida compará-los com dados experimentais de altura de maré observado no referido porto e os dados de maré obtidos pelo software "Sistema Base de Hidrodinâmica Ambiental" (SisBAHIA 9.0) (ROSMAN, 2015). Dados de PM e BM foram retirados do site da DHN e inseridos em um arquivo que, posteriormente, são 
lidos por um código desenvolvido em linguagem FORTRAN 2003, que calcula as alturas de maré em instantes entre PM e BM.

\section{MATERIAL E MÉTODOS \\ Medições experimentais da maré no porto de Belém-PA}

As medições in loco dos dados de alturas de marés foram executadas usando uma régua linimétrica, instalada de acordo com o zero hidrográfico e localizada no Porto de Belém, as margens da Bahia do Guajará no município de Belém-PA. A figura 1 mostra a localização exata da régua no porto. A régua linimétrica se localiza próxima a uma plataforma flutuante de praticagem de barra do porto de Belém, possuindo as seguintes coordenadas geográficas $01^{\circ} 26^{\prime} 10.64^{\prime \prime} \mathrm{S}$ e $048^{\circ} 2^{\circ}$ ' 38.20" W.

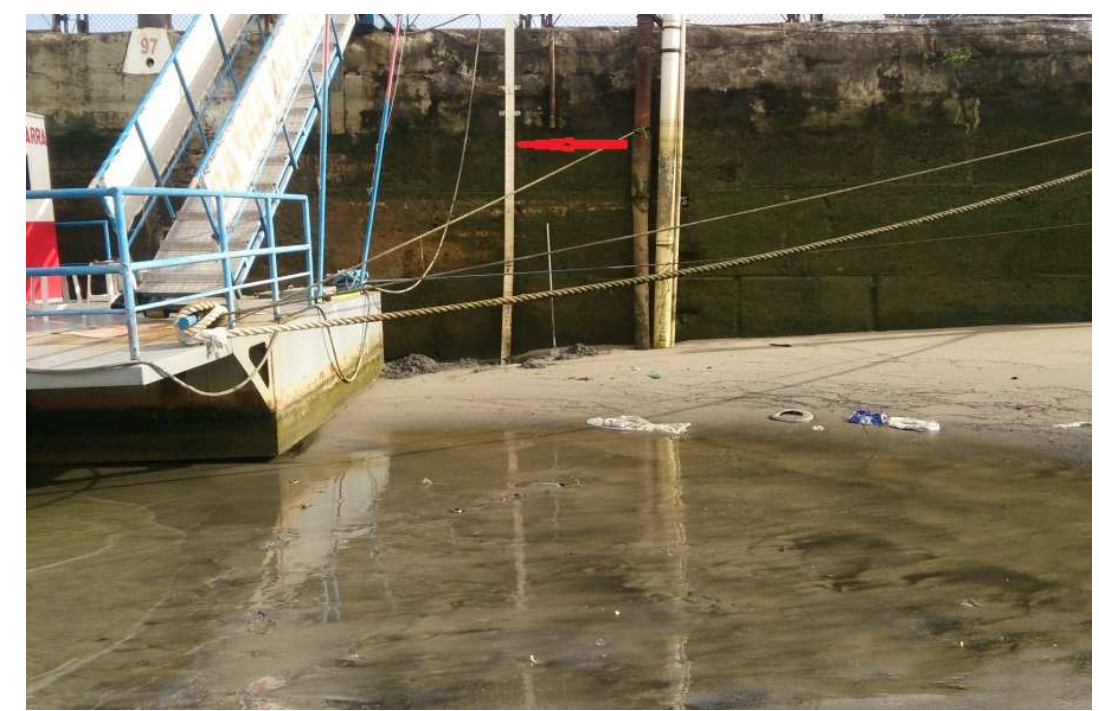

FIGURA 1 - Localização da régua linimétrica no Porto de Belém.

Fonte: Os autores, (2016).

Os dados de maré foram registrados em intervalos de cinco minutos por meio de observação visual na régua linimétrica. A medição se dividiu em dois momentos, uma medição para estação menos chuvosa (08/09/2015) e a outra para estação mais chuvosa (11/02/2016). Isto foi necessário devido as condições meteorológicas serem bastante distintas no período chuvoso e não chuvoso na Amazônia, o que certamente afetará as condições da maré e consequentemente suas alturas.

\section{Cálculo das alturas de maré usando o Software SisBaHia 9.0}

O SisBAHIA é um sistema de modelos computacionais registrado pela Fundação Coppetec, órgão gestor de convênios e contratos de pesquisa da COPPE/UFRJ - Instituto Aberto Luiz Coimbra de Pós-Graduação e Pesquisa de Engenharia (COPPE) da Universidade Federal do Rio de Janeiro. Neste trabalho o SisBAHIA foi usado para calcular os níveis de maré no porto de Belém e comparar os resultados com os dados experimentais e os dados calculados pela ICH. Para isso, o módulo de previsão do software foi acionado e dados das constituintes harmônicas de níveis de maré no porto de Belém foram inseridos de acordo com o Quadro 1. Após os dados das harmônicas serem inseridos no software, previsões de 
valores de altura de maré em séries temporais a intervalos definidos pelo usuário foram obtidos.

QUADRO 1: Constantes harmônicas referentes à estação maregráfica do porto de Belém.

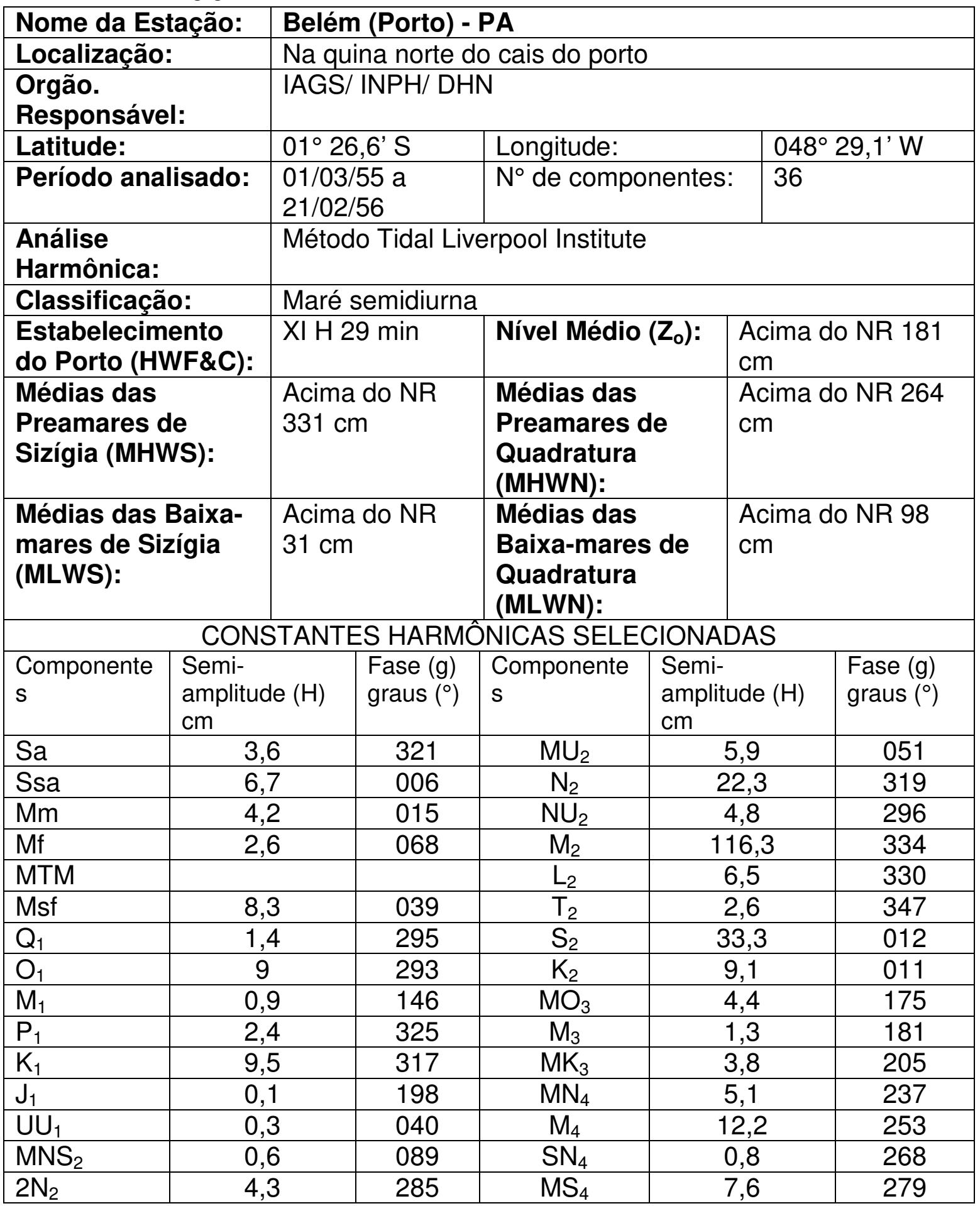

Fonte: FEMAR, (2016).

Cálculo da altura de maré usando a Interpolação Cúbica Hermitiana

De acordo com os trabalhos de ARAÚJO (2001), PROGÊNIO et al. (2015) e OLIVEIRA et al. (2015), a interpolação hermitiana usa o valor da variável (П) e de 
seus gradientes locais $\left(\eta^{\prime}=\partial \eta / \partial t\right)$ para valores fornecidos da variável independente (t). As equações usadas se apresentam como:

$$
\begin{aligned}
& \eta=N 1 \eta 1+N 2 \eta 1^{\prime}+N 3 \eta 2+N 4 \eta 2{ }^{\prime} \\
& N 1=1-3(t / T)^{2}+2(t / T)^{3} \\
& N 2=t+T\left[(t / T)^{3}-2(t / T)^{2}\right] \\
& N 3=3(t / T)^{2}-2(t / T)^{3} \\
& N 4=t+T\left[(t / T)^{3}-2(t / T)^{2}\right]
\end{aligned}
$$

Onde N1, N2, N3 e N4 são funções de interpolação cúbicas hermitianas, n a altura da maré, T intervalo de tempo e t o tempo. De acordo com ARAúJO (2001), tomando-se $\eta 1$ e $\eta 2$ como valores associados nas extremidades de um intervalo de tempo T correspondente à maré alta e baixa respectivamente, suas derivadas $\eta 1^{\prime} \mathrm{e}$ ฤ2' tornam-se nulas, obtendo-se a equação (6) para estimativa da altura de maré (h) em qualquer instante intermediário:

$h=N 1 \eta 1+N 3 \eta 2$

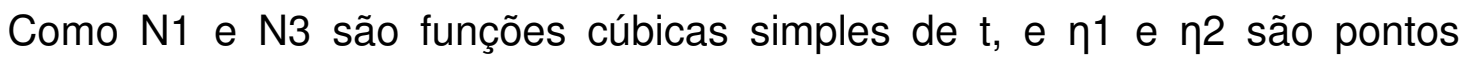
sucessivos de inflexão conhecidos em $\mathrm{T}$, estima-se facilmente $\mathrm{h}$ por meio de (6) (ARAÚJO, 2001). A utilização deste procedimento para aproximação do cálculo da altura de maré é razoável, tendo em vista que, a curva de variação da maré obedece aproximadamente uma função cúbica.

Um código em linguagem FORTRAN 2003 foi desenvolvido com objetivo de implementar as equações (2), (4) e (6). Os valores de BM e PM, referente aos dias 08/09/2015 e 11/02/2016, foram retirados da tábua de maré (DHN, 2016), conforme mostra a tabela 1 Tais valores são inseridos no procedimento de interpolação e o resultado para a estimativa de altura de maré entre estes intervalos são calculados pelo código desenvolvido.

TABELAS 1 - BM e PM disponíveis na tabua das Marés do Porto de Belém nos dias 08/09/15 e 11/02/16.

\begin{tabular}{|c|c|c|}
\hline Dia & Hora & Altura de Maré $(\mathbf{m})$ \\
\hline TER 08/09/2015 & $00: 58$ & $\mathrm{BM}=1.1$ \\
\hline & $07: 21$ & $\mathrm{PM}=2.8$ \\
\hline & $15: 28$ & $\mathrm{BM}=0.8$ \\
\hline & $20: 30$ & $\mathrm{PM}=2.8$ \\
\hline QUI 11/02/2016 & $01: 08$ & $\mathrm{PM}=3.6$ \\
\hline & $08: 51$ & $\mathrm{BM}=0.1$ \\
\hline & $13: 23$ & $\mathrm{PM}=3.5$ \\
\hline & $20: 53$ & $\mathrm{BM}=0.2$ \\
\hline
\end{tabular}

Fonte: Os autores, (2016).

A figura 2 mostra o algoritmo do código desenvolvido no presente trabalho. $\mathrm{O}$ funcionamento básico do algoritmo consiste na entrada dos valores de BM e PM e seus respectivos tempos instantâneos de ocorrência. De posse destes dados calcula-se o intervalo de tempo $\mathrm{T}$, que corresponde ao tempo entre uma BM e uma $\mathrm{PM}$, ou vice-versa. Assume-se um índice jt que conta o tempo de um minuto em um minuto até o intervalo de tempo T. Com o contador temporal definido, calcula-se os polinômios interpoladores N1 e N3 definidos respectivamente pelas equações (2) e (4) e consequentemente a estimativa $h$ de altura de maré. Por fim, os valores de 
saída correspondentes ao tempo e altura de maré são apresentados conforme configuração do usuário.

$$
\begin{aligned}
& \text { Início algoritmo } \\
& \text { ler t1, t2, n1, n2 } \\
& \text { calcular } \mathrm{T}=\mathrm{t} 2-\mathrm{t} 1 \\
& \text { para jt de } 1 \text { até } T \text { faça } \\
& \mathrm{N} 1=1-3(\mathrm{t} / \mathrm{T})^{2}+2(\mathrm{t} / \mathrm{T})^{3} \\
& \mathrm{~N} 3=3(\mathrm{t} / \mathrm{T})^{2}-2(\mathrm{t} / \mathrm{T})^{3} \\
& \mathrm{~h}=\mathrm{N} 1 \mathrm{n} 1+\mathrm{N} 3 \mathrm{n} 2
\end{aligned}
$$

\section{Comparação e potencialidade da ICH}

\section{RESULTADOS E DISCUSSÃO}

O código utilizado no presente trabalho foi desenvolvido em linguagem FORTRAN 2003 e implementado em um PC com processador core i3 e 1,7 GHz de velocidade de processamento. Os resultados dos cálculos das alturas de marés obtidos com o código foram comparados com os dados experimentais e os calculados pelo SisBAHIA nos dias 08/09/2015 e 11/02/2016, conforme observado nas figuras 3 e 4 .

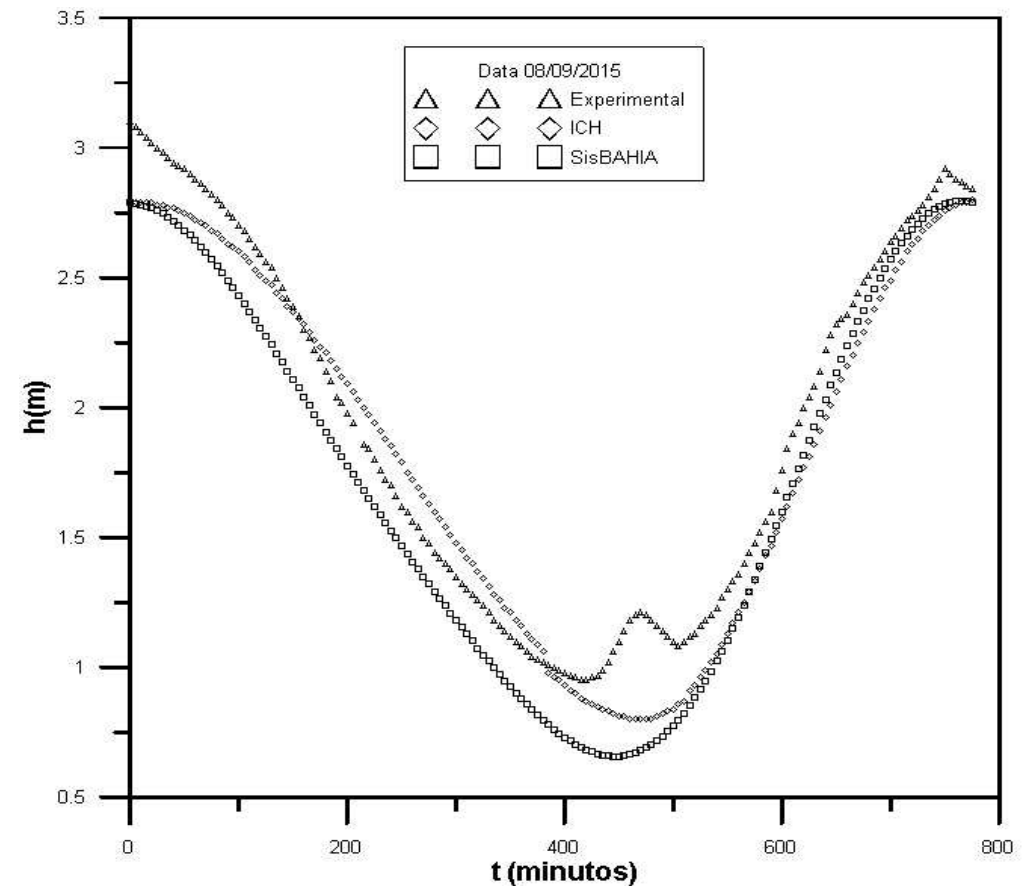

FIGURA 3 - Resultados das simulações e experimento no dia 08/09/15.

Fonte: Os autores, (2016). 


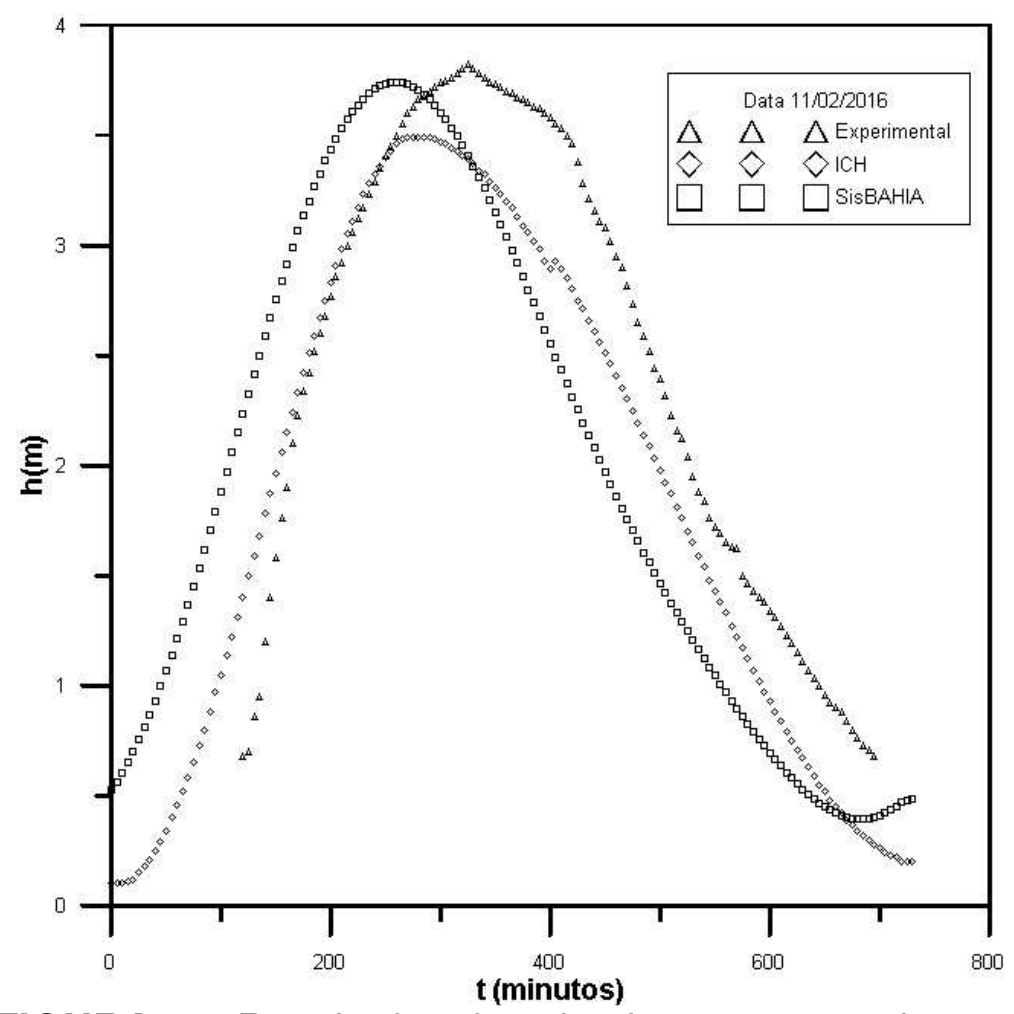

FIGURA 4 - Resultados das simulações e experimento no dia 11/02/16.

Fonte: Os autores, (2016).

Observa-se na figura 3 o mesmo comportamento físico entre os resultados obtidos pela Interpolação Cúbica Hermitiana $(\mathrm{ICH})$, os resultados obtidos pelo SisBAHIA e os dados experimentais para os intervalos de 0 a 420 minutos e de 510 a 780 minutos. Salienta-se que este comportamento físico da maré, verificado no experimento e nas simulações do presente artigo, estão de acordo com os trabalhos de YATES et al. (2013); TSAI \& CHEN (2014) e OLIVEIRA et al. (2015); que mostraram que a elevação e abaixamento de maré têm comportamento sinusoidal. Isto corrobora com os resultados obtidos, mostrando que a ICH simulou adequadamente os níveis de maré no porto em estudo.

Os resultados obtidos no presente trabalho também demonstraram que para os intervalos observados na vazante e enchente da maré no porto de Belém, a ICH se mostrou bastante promissora. Entretanto no intervalo de 420 a 510 minutos os resultados ficaram bastante diferentes das medições. Esta diferença é atribuída a alguns fatores que influenciaram nas medidas experimentais como: pressão atmosférica (CARVALHO \& AMORIM, 2014), vento (KOROTENKO et al., 2012), hidrodinâmica do porto e a proximidade da régua linimétrica com a descarga do canal de água residuária dos bairros de Belém.

Por outro lado, os resultados calculados pelo SisBAHIA para o intervalo de 0 a 420 minutos se apresentaram bons, somente se afastando dos resultados experimentais e da $\mathrm{ICH}$ no início e final do intervalo considerado. No intervalo de 510 a 780 minutos o SisBAHIA mostrou-se também bastante promissor na simulação real da altura de maré. Observou-se neste intervalo uma concordância entre o ICH e o SisBAHIA, mostrando mais uma vez a potencialidade de simulação de níveis de maré pela $\mathrm{ICH}$, tendo em vista que o SisBAHIA é um software bem 
conceituado nacionalmente pela literatura especializada em cálculos hidrodinâmicos (FEITOSA et al., 2013; ROVERSI et al., 2016).

Um fato que também precisa ser ressaltado na explicação do afastamento dos resultados calculados pelo SisBAHIA dos resultados observados é que o software não leva em consideração a maré meteorológica, ou seja, se baseia apenas em fenômenos astronômicos. A maré meteorológica é responsável também pelo aumento ou diminuição do nível do mar devido as condições climáticas num dado local (DUTRA et al., 2014). Apesar da provável influência dos ventos e da pressão atmosférica na dinâmica das águas no porto em análise, não foi objetivo deste trabalho a verificação destas variáveis.

$\mathrm{Na}$ figura 4 também se observa que a $\mathrm{ICH}$ apresentou 0 mesmo comportamento físico dos dados experimentais e do SisBAHIA, principalmente, entre os intervalos 100 a 270 minutos e entre 420 e 700 minutos. Fato marcante na figura 4 é o afastamento significativo dos resultados obtidos pelo SisBAHIA dos resultados experimentais, mostrando que na estação mais chuvosa o SisBAHIA não se adequou à previsão dos níveis de preamar e baixamar. Isto mais uma vez demonstra a potencialidade do uso do ICH em comparação com o SisBAHIA para as medições de maré no porto de Belém, pois os resultados se mostraram mais próximos da realidade.

Em termos gerais há diferenças observadas; tanto na amplitude quanto na defasagem entre as marés simuladas e a medida. Embora, isto em primeira análise represente um certo desconforto, a potencialidade da ferramenta apresentada no presente trabalho se mostra eficaz nas duas situações físicas analisadas, ou seja, estação menos chuvosa com maré vazando até atingir a BM e depois novamente enchendo (figura 3) e estação mais chuvosa com maré enchendo até atingir a PM e em seguida vazando (figura 4).

\section{Análise de erro}

Com objetivo de verificação da qualidade dos resultados obtidos pela ICH e pelo SisBAHIA, uma análise de erro relativo em relação a medição experimental da maré foi conduzida. A tabela 2 mostra os valores máximos, mínimos e médios dos erros relativos.

TABELA 2 - Análise dos erros máximo, mínimo e médio das simulações.

\begin{tabular}{l|c|c|c}
\hline ICH em relação à maré medida & Mínimo (\%) & Maximo (\%) & Média (\%) \\
\hline $08 / 09 / 2015$ & 0,42 & 33,88 & 9,32 \\
\hline $11 / 02 / 2016$ & 0,29 & 114,28 & 21,60 \\
\hline $\begin{array}{l}\text { SisBaHiA em relação a maré } \\
\text { medida }\end{array}$ & Mínimo (\%) & Maximo (\%) & Média (\%) \\
\hline $08 / 09 / 2015$ & 1,69 & 44,08 & 13,81 \\
\hline $11 / 02 / 2016$ & 0,10 & 232,18 & 38,57 \\
\hline
\end{tabular}

Fonte: Os autores, (2016).

Analisando a tabela 2, observa-se que os erros relativos mínimo, máximo e médio cometidos no cálculo das variações de marés utilizando a $\mathrm{ICH}$ são em geral menores que os erros cometidos pelo SisBAHIA, tanto no período chuvoso quanto não chuvoso, excetuando o erro mínimo observado no dia 11/02/2016. Isso mostra que a ferramenta apresentada neste trabalho consegue prever de maneira satisfatória as elevações e abaixamentos dos níveis de maré no porto de Belém mantendo um erro médio de cálculo dentro de um patamar razoável. 
O código desenvolvido apresenta médias de erro relativo menores para estação menos chuvosa, logo, este resultado mostra que é necessária a inserção de fatores climáticos no código, o que aumentaria a precisão. Outro fato que deve ser levado em consideração nos erros experimentais observados no presente trabalho é a precisão da régua linimétrica. Segundo a ANA (2014), este tipo de medição não é - mais recomendado quando se deseja precisão dos resultados, pois sofre constantes influências da dinâmica do manancial local, além de ser bastante cansativo o processo de medição. Embora tais dificuldades encontradas durante as medições, não se deve esquecer que a régua está instalada de acordo com o zero hidrográfico, que representa as alturas médias alcançadas pelas marés no porto de Belém, o que de certa maneira dá mais credibilidade para as medições realizadas e, portanto, mais precisão no cálculo do erro relativo.

\section{CONCLUSÃO}

Os resultados para altura de maré calculada pelo código apresentaram resultados satisfatórios do ponto de vista físico. No entanto a tentativa de validar o código desenvolvido utilizando-se de medições in loco não apresentou os resultados esperados, fato que pode ser explicado pelas condições adversas encontradas no local de medição e a pouca precisão do instrumento empregado, embora a régua linimétrica estivesse instalada de acordo com o zero hidrográfico. Dessa maneira, para dar maior garantia quanto aos resultados obtidos, julga-se necessário a validação utilizando-se meios de medição mais precisos, onde os erros sejam minimizados, tais como uso de sonar, que possui precisão de medição da ordem de 0,005 a $0,01 \mathrm{~m}$, baixo custo e pouca manutenção. Além disso, como o código desenvolvido via Fortran 2003 limita-se a simular apenas a maré astronômica, é recomendado considerar outros fatores, como pressão atmosférica, vento e precipitação pluviométrica na formação da maré meteorológica.

A medição experimental foi realizada em apenas um ciclo de maré para cada estação, logo, esta pode não ser suficiente para se estabelecer uma comparação entre a simulação e os dados experimentais mais precisa. Desta forma é necessário realizar mais medições experimentais para que se possa obter um melhor cenário do comportamento da maré no Porto de Belém.

Os resultados obtidos pela $\mathrm{ICH}$ se aproximaram mais das medições da maré real, tanto para a estação mais chuvosa quanto menos chuvosa, do que as simulações produzidas pelo SisBaHiA. Logo, estes resultados indicam que o código é promissor, garantindo uma boa aproximação da previsão do fenômeno natural da maré astronômica. Ressalta-se que os bons valores encontrados pela ICH reforçam sua utilização em estudos de variação nos níveis de maré na costa Brasileira.

\section{REFERÊNCIAS}

AGÊNCIA NACIONAL DE ÁGUAS - ANA. Medição de descarga liquida em grandes rios - Manual técnico. $2^{\circ}$ Edição, Brasília, DF, 2014, 94 p.

ARAÚJO, A.M. Síntese de Maregramas a Partir de Previsões de Maré, 2001. Disponível em: <https://www.researchgate.net/publication/267371629>. Acesso em: 18 de Abril de 2016.

ARAQUISTAIN, T. M. Tidal Power: Economic and Technological assessment. Department of Thermal Engineering, Tsinghua University, 2010. 
CHARLIER, R. H.; FINKL, C. W. Ocean Energy: Tide and Tidal Power. German, Springer-Verlag, 2009, 270 p., ISBN: 978-3-540-77931-5, e-ISBN: 978-3-540-779322, doi 10.1007/978-3-540-77932-2.

CLARK, R. H. Elements of Tidal-Electric Engineering. USA, John Wiley \& Sons, 2007, 289 p., ISBN 978-0-470-10709-6.

CUOMO, S.; GALLETTI, A.; GIUNTA, G.; MARCELLINO, L. Piecewise Hermite interpolation via barycentric coordinates. Ricerche di Matematica, v. 64, n. 2, p. 303-319, 2015. doi:10.1007/s11587-015-0233-0

CARVALHO, L. R. M.; AMORIM, H. S. Observando as marés atmosféricas: uma aplicação da placa Arduino com sensores de pressão barométrica e temperatura. Revista Brasileira do Ensino de Física, v. 36, n. 3, 2014, disponível em: < http://dx.doi.org/10.1590/S1806-11172014000300013>. Acesso em: 20 de dezembro de 2016.

DHN, Diretoria de Hidrografia e Navegação da Marinha do Brasil. Previsão de marés. Informação. 2016. Disponível em: <http://www.mar.mil.br/dhn/chm/boxprevisao-mare/tabuas/index.htm>. Acesso em: 19 de março de 2016.

DUTRA, F. R. L. S.; CIRANO, M.; BITTENCOURT, A. C. S. P.; TANAJURA, C. A. S.; LIMA, M. Meteorological tides and episodes of severe coastal erosion on the coast of salvador, Bahia state, Brazil. Revista Brasileira de Geofísica, v. 32, n. 4, 2014. doi: http://dx.doi.org/10.22564/rbgf.v32i4.551

FEITOSA, R. C.; ROSMAN, P. C. C.; BLENINGER, T.; WASSERMAN, J. C. Coupling bacterial decay and hydrodynamic models for sewage outfall simulation. Journal of Applied Water Engineering and Research, v. 1, n. 2, p.137-147, 2013. Disponível em: <http://dx.doi.org/10.1080/23249676.2013.878882>. Acesso em 20 de dezembro de 2016.

FEMAR, Fundação de Estudos do mar. Catálogo de estações maregráficas brasileiras 2016.2 Disponível em: <https://fundacaofemar.org.br/biblioteca/emb/tabelas/054.html>. Acesso em: 12 de março de 2016.

KOROTENKO, K. A.; SENTCHEV, A. V.; SCHMITT, F. G. Effect of variable winds on current structure and Reynolds stresses in a tidal flow: analysis of experimental data in the eastern English Channel. Ocean Science, v. 8, n. 6, p. 1025-1040, 2012, doi:10.5194/os-8-1025-2012.

LEITE NETO, P. B; SAAVEDRA, O. R; RIBEIRO, L. A. S. Optimization of electricity generation of a tidal power plant with reservoir constraints. Renewable Energy, v. 81, p.11-20, 2015.disponível em:<http://dx.doi.org/10.101016/j.renene.2015.03.011>. doi:10.101016/j.renene.2015.03.011

OLIVEIRA, L. L. P; BLANCO, C. J. C; MESQUITA, A. L. A. Metodologia para projetos de centrais maremotrizes, Revista Ciência e Tecnologia, v.18, n.33, p.55-64, 2015. 
Disponível em: <http://www.revista.unisal.br/sj/index.php/123/article/view/452>. Acesso em 19 de junho de 2016.

PROGENIO, M. F.; BARBOSA JUNIOR, R. N. S.; SOUZA, M. J. R. Ferramenta Matemática Alternativa para Cálculo de maré Astronômica. In: $4^{\circ}$ simpósio de estudos e pesquisas em ciências ambientais na Amazônia, 3, 2015. Anais. BelémPA, 2015, p. 123 - 132 . Disponível em: < http://www4.uepa.br/paginas/pcambientais/simposio/anais_artigos_vol_3_simposio_ 2015.pdf >. Acesso em: 19 de abril de 2016.

ROSMAN, P. C. C. (Org.). Referência técnica do SisBaHiA® - Sistema Base de Hidrodinâmica Ambiental. Rio de Janeiro: COPPE/UFRJ, 2015.

ROVERSI, F.; ROSMAN, P. C. C.; HARARI, J. Análise da renovação das águas do Sistema Estuarino de Santos usando modelagem computacional. Revista Ambiente \& Água, v. 11, n. 3, 2016, doi:10.4136/ambi-agua.1770.

TSAI, JIA-SHIUAN; CHEN, F. The Conceptual Design of a Tidal Power Plant in Taiwan. Journal of Marine Science and Engineering, v. 2, p. 506-533, 2014, doi:10.3390/jmse2020506.

YATES, N.; WALKINGTON, I.; BURROWS, R.; WOLF, J. Appraising the extractable tidal energy resource of the UK's western coastal Waters. Philosophical Transactions of the Royal Society A 371 20120181, 2013. Disponível em: <http://dx.doi.org/10.1098/rsta.2012.0181 >. Acesso em 25 de agosto de 2015. 